\title{
Squamous cell carcinoma: from precursor lesions to high-risk variants
}

\author{
Bruce R Smoller
}

\author{
Department of Pathology, University of Arkansas for Medical Sciences, Little Rock, AR, USA
}

\begin{abstract}
Cutaneous squamous cell carcinoma is second only to basal cell carcinoma in its incidence within our population. It is among the most common types of neoplasm afflicting the human race. Most cases are related to exposure to sunlight. This initial portion of the chapter will focus upon the biologic progression that results in the ultimate development of fully formed squamous cell carcinoma. The latter portion will focus upon the histologic features of squamous cell carcinoma, which may be potential prognostic indicators. While pathologists currently provide clinicians with information about many histologic features that may affect the outcome for patients with melanoma, there is little impetus for similar behavior on the part of pathologists when diagnosing squamous cell carcinomas. This is largely due to the relatively low metastasis rate for these tumors compared with melanomas; however, it may be possible for pathologists to separate out those squamous cell carcinomas with a higher chance for recurrence and metastasis. This chapter will examine many of these histologic features and propose a rationale for including this information in pathology reports.

Modern Pathology (2006) 19, S88-S92. doi:10.1038/modpathol.3800509
\end{abstract}

Keywords: squamous cell carcinoma; actinic keratosis; prognosis; histology

Cutaneous squamous cell carcinoma is the second most frequent malignancy in human beings (trailing only basal cell carcinoma). Recent reports have suggested that, in fact, squamous cell carcinoma may be a more frequent neoplasm than even basal cell carcinoma depending upon how one defines this tumor (see below). In most cases, the diagnosis is a straightforward one that does not pose major diagnostic difficulties for the pathologist. However, the distinction from actinic keratosis can be difficult and the criteria for distinguishing between the two entities are controversial.

Actinic (solar) keratoses are very common lesions that occur on sun-damaged skin. The clinical features include erythematous patches or plaques with increased scale. In some cases, ulceration may be present, though this is probably secondary to excoriation in most cases. Telangiectasias are often present.

The histologic features of actinic keratosis have been well described. The epidermis demonstrates parakeratosis that may be confluent or intermittent. In early lesions, there is generally focal sparing, with orthokeratosis of the stratum corneum directly

Correspondence: Dr BR Smoller, MD, Department of Pathology, University of Arkansas for Medical Sciences, 4300 W Markham, Little Rock, AR, USA.

E-mail: smollerbrucer@uams.edu

Received 14 September 2005; accepted 29 September 2005 overlying the outflow tracts of the cutaneous appendages. The epidermis may be atrophic, of normal thickness, or acanthotic. In most cases, there is diminution of the granular layer in areas underlying the parakeratosis (Figure 1).

The acanthotic or hyperplastic variant is characterized by elongated rete ridges, but not by a general thickening of the entire epidermis. As the lesions become increasing hyperplastic, there is a tendency to form downward protrusions, resembling rete ridges. These are markedly increased over the normal frequency of the rete ridge pattern and are a helpful feature in arriving at the diagnosis in some cases. The pathognomonic feature is that of cytologic atypia of keratinocytes. The atypia begins within the basal layer of the epidermis. The keratinocytes are larger than normal basal keratinocytes and lose their polarity. The nuclei may be vesicular or hyperchromatic, but the nuclear:cytoplasmic ratio is altered compared with the normal keratinocytes. The normal maturation sequence is disrupted as the lesions become more advanced, with keratinocyte atypia extending above the basal layer (Figure 2).

Most dermatopathologists distinguish between actinic keratosis and squamous cell carcinoma in situ based upon the upward extent of the keratinocyte dysmaturation. When the atypia involves keratinocytes throughout the full thickness of the epidermis, the lesion is designated as a squamous cell carcinoma (in situ), while atypia that falls 


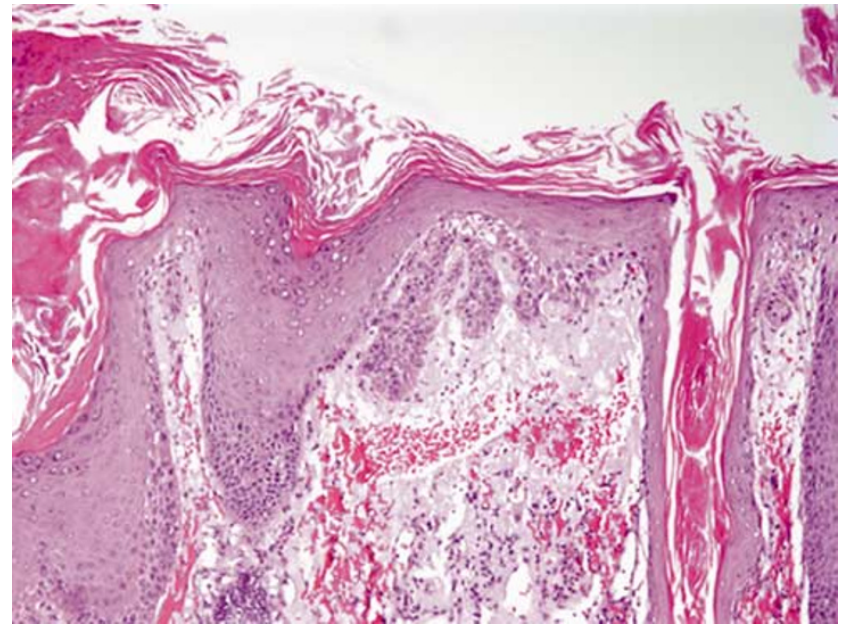

Figure 1 Actinic keratosis demonstrating alternating parakeratosis and orthokeratosis, elongated rete ridges, marked solar elastosis, and atypia of basal keratinocytes.

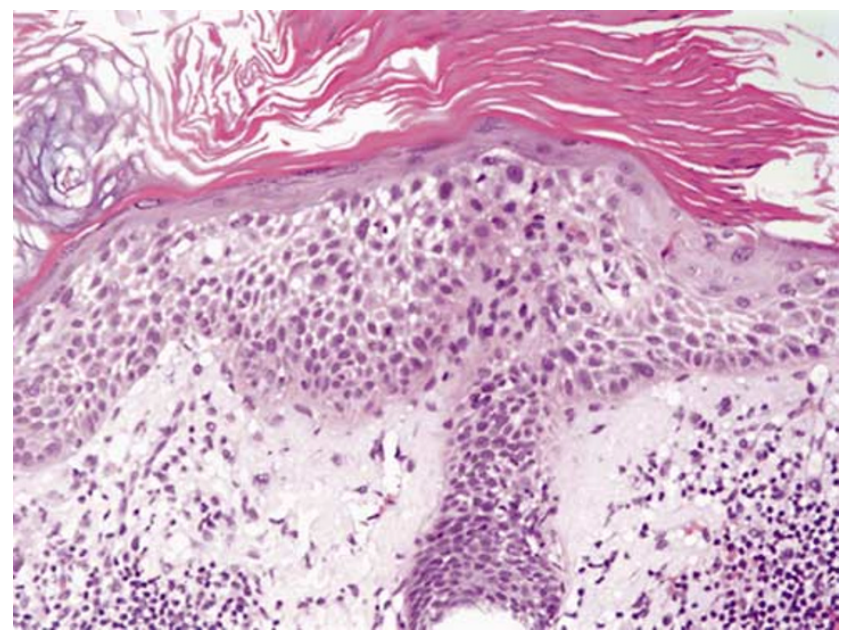

Figure 2 Cytologic atypia is present within keratinocytes from the basal layer up to the granular layer. There is lack of organized maturation and a haphazard arrangement to the cells, characteristic of actinic keratosis.

short of this retains the diagnosis of actinic keratosis. This distinction is where the controversy persists in the literature. ${ }^{1,2}$ In some cases, there is marked acantholysis, as the deranged keratinocytes fail to form functional desmosomes. Individual keratinocyte necrosis is also seen in some cases.

As actinic keratoses are the result of extensive ultraviolet light exposure, there is evidence of this process within the underlying dermis. A prerequisite for making such a diagnosis includes solar elastosis within the superficial dermis. This is most commonly accompanied by telangiectasia of vessels within the superficial vascular plexus.

Completing the histologic changes, an inflammatory response is seen as a reaction to most actinic keratoses. The infiltrate is comprised mainly of lymphocytes and histiocytes, though plasma cells are not infrequent. The infiltrate may be relatively sparse or very brisk and lichenoid, obscuring the dermal epidermal junction. The degree and quality of the immune response do not appear to correlate with the degree of cytologic atypia or with invasion into the dermis (progression to squamous cell carcinoma), but, rather, appears to be a function of the individual host.

It is virtually impossible to ascertain an incidence rate for actinic keratoses, as most of these lesions do not come to medical attention. Even among patients who do seek medical intervention, the vast majority of these neoplasms are not submitted for histologic examination, but, rather, are treated with locally destructive therapies including cryosurgery, electrodessication and curettage or topical chemotherapies. There have been many reports in the literature that attempt to arrive at some type of incidence rate, but these studies have routine failed to provide complete clinical and histologic confirmation and significant long-term follow-up.

It has been argued by some that actinic keratosis and squamous cell carcinoma are two names for the same process. This opinion rests upon the data that in most cases of squamous cell carcinoma developing on sun-exposed skin, adjacent actinic keratosis is found. In addition, the cytologic features of the involved cells are identical, the cytogenetic aberrations are identical, and the epidemiologic characteristics the same. The argument continues that squamous cell carcinomas do not develop de novo, but rather develop along a spectrum of atypia. Further, it is not uncommon to see foci of unequivocal dermal invasion by atypical keratinocytes in lesions that have not yet attained full-thickness epidermal atypia. These types of tumors do not appear to be progressing through an orderly stepwise process, but rather appear to progress directly from early basilar atypia directly to invasive squamous cell carcinoma.

However, I believe that the counterarguments are stronger. It has been argued that the evolution of squamous cell carcinoma of the skin shares some features with that of squamous cell carcinoma of the cervix. In the cervix, there is a well-recognized spectrum ranging from CINI to CINIII. In this system, it is firmly established that only a minority of CINI lesions progress to CINII, and still a lesser minority progresses to CINIII. Only a small percentage of these lesions eventually become invasive squamous cell carcinomas.

The rate of progression from actinic keratosis to squamous cell carcinoma is difficult to assess for the reasons stated above. ${ }^{3-6}$ Estimates range from less than one in thousand to $10 \%$, depending upon the criteria used. In no circumstances, however, is it believed that the majority of lesions, even if left completely untreated, will eventuate in invasive squamous cell carcinoma. In fact, there are some studies that document spontaneous regression of 
actinic keratoses that are left untreated. ${ }^{5,7}$ It is not possible at this point to determine the exact moment at which an intraepidermal neoplasm acquires the ability to invade the basement membrane, enter the dermis, and eventually to metastasize. Therefore, our abilities as pathologists remain somewhat limited by the tools at our disposal. In keeping with a goal of doing the least harm for patients, it seems prudent to limit our diagnoses of unequivocal carcinoma to those lesions that display either fullthickness intraepidermal atypia (squamous cell carcinoma in situ) or to those that have already invaded into the dermis. Intraepidermal lesions with keratinocyte atypia that extends only part way through the epidermis can safely be regarded as precursor lesions with no risk for metastasis and treated more conservatively.

Once a diagnosis of squamous cell carcinoma has been established, it is important for the pathologist to contribute additional prognostic information to the referring physician. While the overall rate of metastasis is quite low (on the order of $1-2 \%$ ) and difficult to assess precisely (for the same reasons as proffered for actinic keratosis incidence above), this rate is greatly increased in certain circumstances. ${ }^{8-10}$ Traditionally, pathologists have provided clinicians with a template of prognostic indicators when reporting melanomas. This has not been true for squamous cell carcinomas, perhaps in part due to the relatively good prognosis for most of these tumors. Further, analogous prognostic markers have not been studied to the same degree, and this information is, thus, not as readily available to pathologists.

Cytologic characteristics of the neoplastic squamous cells have been studied and reported to be of some prognostic significance in some studies. Among the subtypes frequently separated for analysis include spindle cell squamous cell carcinomas (carcinosarcomas) and acantholytic squamous cell carcinomas. ${ }^{11}$

Neither of these histologic subtypes is associated with any distinct clinical features, though acantholytic tumors are more commonly seen in elderly men with a preference for the head and neck in some series. It has been suggested that, in each of these cases, the presence of cells with these cytologic characteristics may be associated with a higher rate of metastasis and death. However, there is much controversy in the literature regarding these conclusions and many studies have failed to replicate the findings. Therefore, the inclusion of these descriptors within pathology reports may be of limited value to clinicians (Figures 3 and 4).

Early studies attempted to correlate the likelihood of metastasis with cytologic differentiation (Broder's classification; Figure 5). While there was a slight tendency for less differentiated tumors to behave more aggressively, in general, the assessment of differentiation did not correlate with biologic behavior for most neoplasms. Most pathologists do
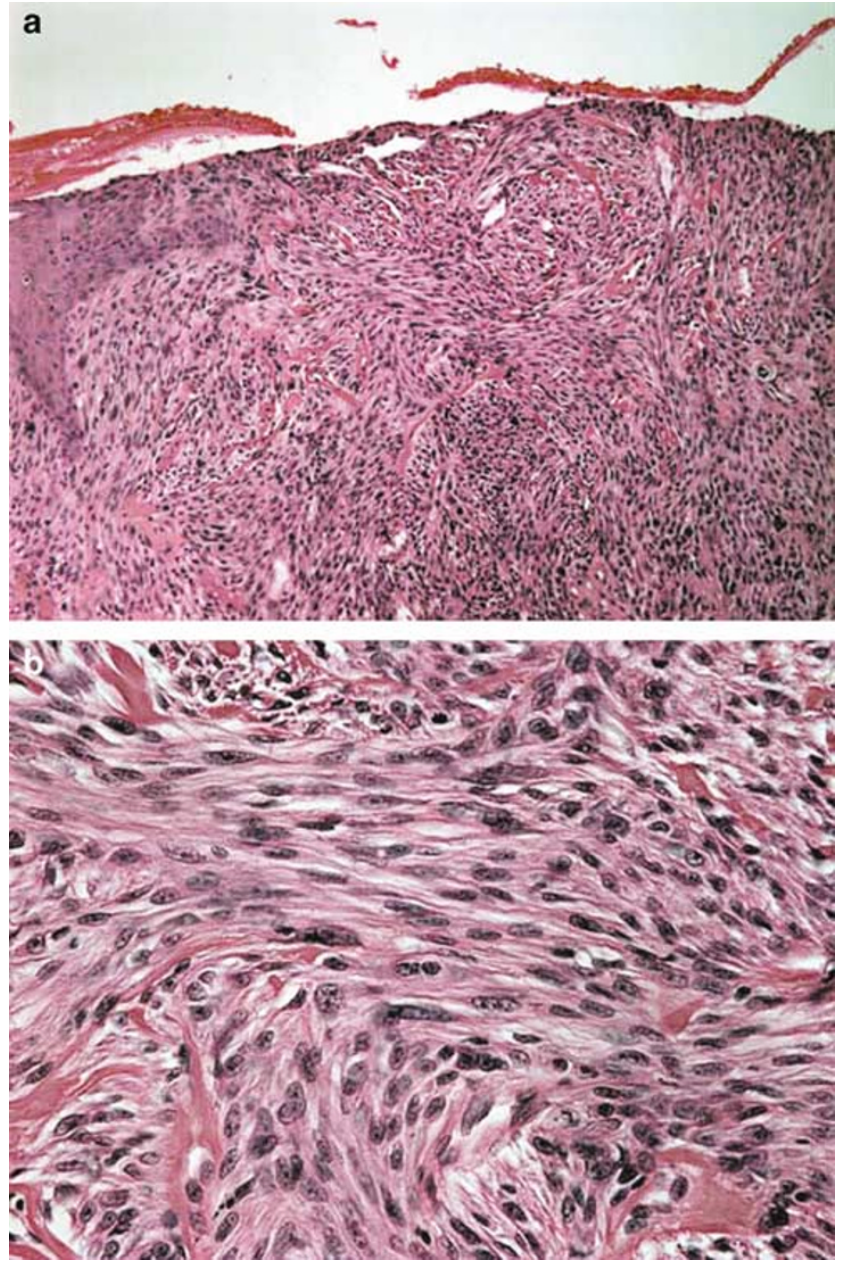

Figure 3 (a) Spindle squamous cell carcinoma growing downward from ulcerated surface. (b) Higher magnification demonstrates atypical spindle-shaped cells coursing in fascicles. Inapparent keratinization and intercellular bridges make immunostains essential in many cases.

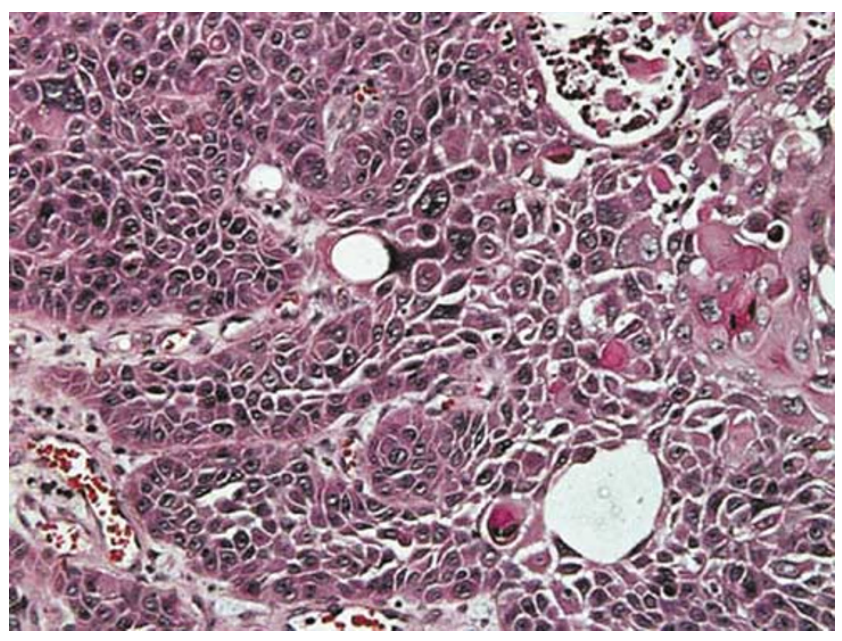

Figure 4 An acantholytic squamous cell carcinoma demonstrates sheets of keratinocytes with foci of acantholysis that resemble ductular differentiation. 


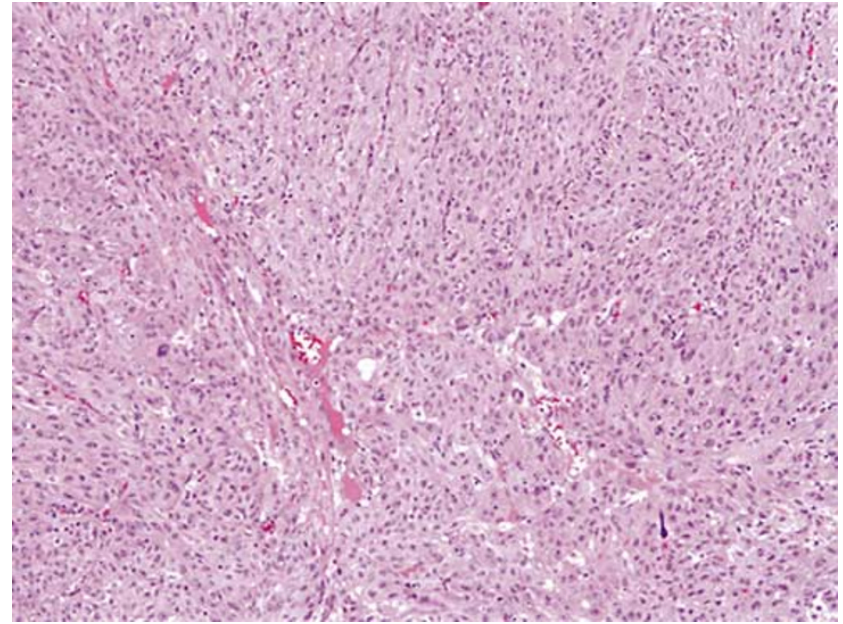

Figure 5 A poorly differentiated squamous cell carcinoma demonstrates marked cellular pleomorphism and virtual absence of keratinization, making a diagnosis of squamous cell carcinoma difficult upon routine histologic sections.

not currently grade the differentiation of cutaneous squamous cell carcinomas.

Prognosis has been related to the site of occurrence in some studies of squamous cell carcinoma. Those occurring on the lips and ears have been shown to have a significantly higher rate of metastasis than those occurring on other sites. ${ }^{12}$ Other relatively high-risk sites of involvement include the penis, scrotum, and anus.

Predisposing conditions also significantly alter the prognosis for cutaneous squamous cell carcinoma. Those neoplasms arising at the site of prior irradiation or at the sites of long-standing ulcers or within osteomyelitic sinus tracts or at sites of previous burn injuries have much higher rates of metastasis than do squamous cell carcinomas arising on actinically damaged skin (Figure 6).

Squamous cell carcinomas that develop in immunosuppressed patients also have higher rates of metastasis. This is especially true for patients who are immunosuppressed following solid organ transplant.

Size has been associated with adverse prognosis in some studies. ${ }^{13-16}$ It has been suggested that larger lesions tend to be more deeply invasive, are often less differentiated, and are more likely to demonstrate vascular and neural invasion. Therefore, it is not clear whether tumor size can be held as an independent prognostic variable for squamous cell carcinoma. While this information is still debatable, it does appear that, for whatever the reason, larger lesions are more likely to result in metastasis and the death of the patient than are small squamous cell carcinomas.

Depth of invasion (Breslow, Clark) has been established as the best predictor of ultimate prognosis for cutaneous melanoma. There have been several studies addressing analogous measurements for cutaneous squamous cell carcinomas. ${ }^{12}$ The data

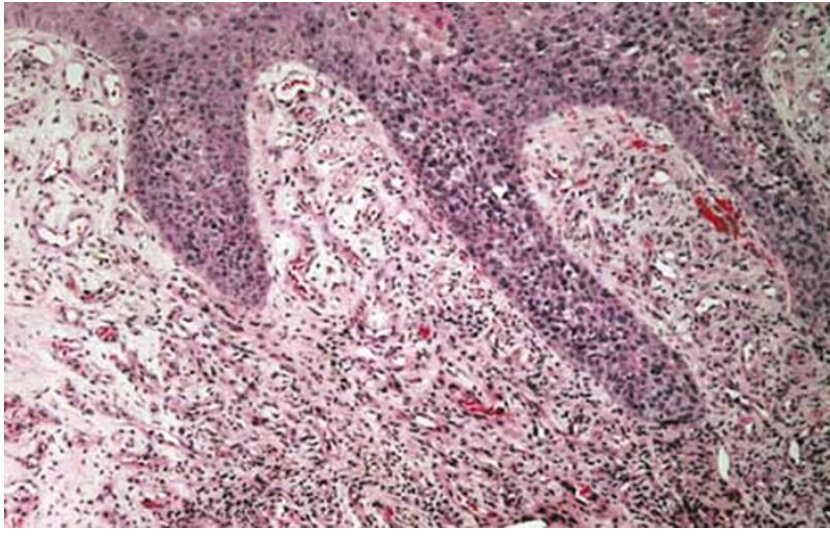

Figure 6 This squamous cell carcinoma is developing within a long-standing stasis ulcer, as evidenced by the extensive proliferation of small, thick-walled vessels within the superficial dermis.

correlating depth of invasion with prognosis has been most thoroughly examined for tumors occurring on the lip. Analogous to the situation with melanoma, actual depth measurements, as well as assessment of level of invasion into the dermis, have been studied as a function of metastasis rate. While the data do seem to support that there is some role for depth measurements for this tumor, most pathologists have not yet incorporated this measurement into common practice schemes. Both the depth measurement and the Clark's level equivalent have been demonstrated to provide additional prognostic information with regard to likelihood of metastasis. In one study, squamous cell carcinomas less than $4 \mathrm{~mm}$ thick were not associated with metastasis in any case. In another study, the metastatic rate was $15 \%$ or tumors thicker than $6 \mathrm{~mm}$, and $4.5 \%$ for tumors between 2 and $6 \mathrm{~mm}$ thick.

The presence of neural and/or vascular invasion has also been reported to be an indicator of adverse prognosis. Perineural invasion may increase the likelihood of local recurrence and was seen much more commonly in tumors that eventually metastasized than in nonmetastasizing controls (Figure 7). Vascular invasion is more difficult to assess and has not yet proven to be of prognostic significance.

The role of a pathologist is to provide clinicians with a diagnosis and with as much prognostic information as possible when examining biopsy material. Towards this end, it may be prudent for pathologists to reconsider the manner in which we report cutaneous squamous cell carcinomas. In a recent survey, despite the fact that most respondents were aware of many of the prognostic indicators described above, the vast majority reported that they did not include many of these features in their reports. ${ }^{17}$ While we are all likely to report many variables in our reporting of melanomas, we tend to neglect these parallel variables with squamous cell carcinomas, almost certainly because of the low likelihood for these tumors to metastasize. While 


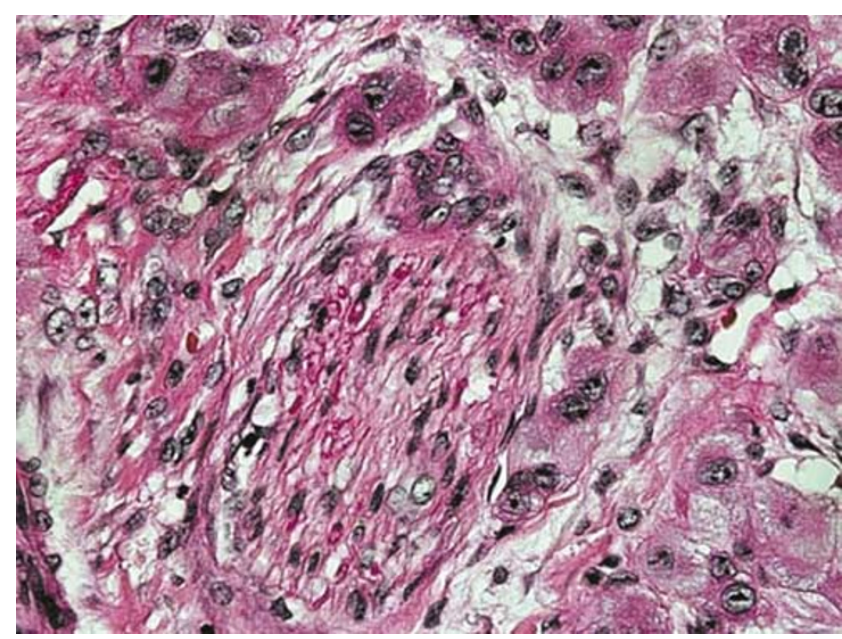

Figure 7 Perineural invasion is seen most frequently at the base of deeply invasive lesions and may portend a worse prognosis.

this is certainly true, there is a small subset of squamous cell carcinomas that do metastasize and result in the death of affected patients. Perhaps as a specialty, we should consider incorporating comments about many of these variables as a routine component of the diagnostic reports of squamous cell carcinomas in hopes of helping clinicians determine immediate treatment and decreasing the numbers of adverse events related to squamous cell carcinomas.

\section{References}

1 Ackerman AB. Solar keratosis is squamous cell carcinoma. Arch Dermatol 2003;139:1216-1217.

2 Lober BA, Lober CW. Actinic keratosis is squamous cell carcinoma. Southern Med J 2000;93:650-657.
3 Marks R, Rennie G, Selwood TS. Malignant transformation of solar keratoses to squamous cell carcinoma. Lancet 1988;8589:795-797.

4 Montgomery H, Dorffel J. Verruca senilis und keratoma senile. Arch Derm Syphilol 1932;166:286-299.

5 Marks R, Foley P, Goodman G, et al. Spontaneous remission of solar keratoses: the case for conservative management. Br J Dermatol 1986;115:649-655.

6 Takemiya M, Ohtsuka H, Miki Y. The relationship between solar keratoses and squamous cell carcinomas among Japanese. J Dermatol 1990;17:342-346.

7 Thompson SC, Jolley D, Marks R. Reduction of solar keratoses by regular sunscreen use. N Engl J Med 1993;329:1147-1151.

8 Lund HZ. How often does squamous cell carcinoma of the skin metastasize? Arch Dermatol 1965;92:635-637.

9 Moller R, Reymann F, Hou-Jensen K. Metastases in dermatological patients with squamous cell carcinoma. Arch Dermatol 1979;115:703-705.

10 Callen JP, Bickers DR, Moy RL. Actinic keratoses. J Am Acad Dermatol 1997;36:650-653.

11 Evans HL, Smith JL. Spindle cell squamous cell carcinoma and sarcoma-like tumors of the skin: a comparative study of 38 cases. Cancer 1980;45:26872697.

12 Frierson Jr HF, Cooper PH. Prognostic factors in squamous cell carcinoma of the lower lip. Hum Pathol 1986;17:346-354.

13 Johnson TM, Rowe DE, Nelson BR, et al. Squamous cell carcinoma of the skin (excluding lip and oral mucosa). J Am Acad Dermatol 1992;26:467-484.

14 Breuninger H, Black B, Rassner G. Microstaging of squamous cell carcinomas. Am J Clin Pathol 1990;94:624-627.

15 Friedman HL, Cooper PH, Wanebo HJ. Prognostic and therapeutic use of microstaging of cutaneous squamous cell carcinoma of the trunk and extremities. Cancer 1985;56:1099-1105.

16 Kwa RE, Campana K, Moy RL. Biology of cutaneous squamous cell carcinoma. J Am Acad Dermatol 1992;26:1-26.

17 Khanna M, Fortier-Riberdy G, Dinehart SM, et al. Histopathologic evaluation of cutaneous squamous cell carcinoma: results of a survey among dermatopathologists. J Am Acad Dermatol 2003;48:721-726. 